\title{
P 140 IMPROVING COMMUNICATION CONCERNING USE OF POTENTIALLY SEDATIVE INJECTABLE MEDICATIONS AT END OF LIFE
}

Kathryn Richardson, ${ }^{1}$ Ruth Hiscock, ${ }^{2}$ Corinna Midgley'. 'Saint Francis Hospice, Romford, England; ' GP Registrar, Weston Road Surgery, Romford, England

10.1136/bmjspcare-2014-000654.181

Aims To explore use of potentially sedative injectable medicines at end of life in a hospice inpatient population, and ensure good quality of communication surrounding the use of such drugs. A retrospective case notes audit of people who had recently died in the hospice was developed. Frequency and dose range of injectable sedatives used in the last week of life were noted, also range of symptoms recorded, documentation of intent for those medications. and that discussion with patient/family concerning intent/ potentially sedating side effect had been achieved, with the plan of care agreed.

Results 46/50 notes were analysed (sequential deaths - 4 not found). 42 were on an end of life care pathway when they died. Use of medicines which could potentially sedate was high: medicines reviewed were midazolam, levomepromazine and phenobarbitone. Only 3 were on no such medication. 40/46 were receiving medicine via an infusion. Midazolam and levomepromazine median/mode doses were low. Intention was better documented than in a previous audit but there were clear gaps in documentation concerning discussion with patient, family and team. In the 5 for whom the intent was sedation there was good documentation concerning discussion with a senior doctor - but poor documentation of discussion with patient/family/team.

Conclusions The audit identified improvement since intention/ counselling had become part of syringe driver policy, but unclear patient, family and team engagement in the plan of care. A policy on use of potentially sedative medications has now been developed to better support good communication concerning such drugs. MDT teaching has engaged staff in the importance of such communication. Key recommendations for use of sedation in palliative care (based on the European Association for Palliative Care, 2007) are now included. The policy has been positively received by staff (feedback included in this review) and is now in use. 